\title{
Memory for locations within regions: Spatial biases and visual hemifield differences
}

\author{
B. LAENG, M. PETERS, and B. McCABE \\ University of Guelph, Guelph, Ontario, Canada
}

\begin{abstract}
Memory for location of a dot inside a circle was investigated with the circle in the center of a computer screen (Experiment 1) or with the circle presented in either the left or the right visual field (Experiment 2). In both experiments, as in Huttenlocher, Hedges, and Duncan's (1991) study, the task was to relocate the dot by marking the remembered location. When errors in angular and radial estimates were considered separately, it was found that, in both experiments, the angular locations of estimates of the dots' positions regressed toward different locations inside each quadrant of the circle; the radial locations of the estimates of dots' positions tended to regress toward locations near the circumference. These variations in the direction of bias appeared to reflect a general shift of estimates toward the upper left arc of the circle. The second experiment replicated the preceding effects but also revealed that the regressions within quadrants of angular values were stronger after right visual field than after left visual field presentations. We interpret the dissociation between visual fields as evidence that memory for categorical spatial relations (Kosslyn, 1987) is more dependent on left-hemisphere than on right-hemisphere processing.
\end{abstract}

In situations in which one is required to remember a specific location in which an object may be situated (e.g., Where did I leave my glasses?), we seem to rely on two types of memories of the spatial location. One is based on the memory of an action that relates to the location, such as reaching to/from a certain place, walking to/from a place where a car is parked, or of a situation in which a scene and a location occupied by the object is visualized. Another type of memory about the location of an object seems to be based on information about the regions of space where the object was placed (e.g., we may remember that the glasses were in the bedroom but not exactly where). Regions, however, contain certain areas and specific locations that are more salient than others, due either to the presence of salient landmarks or to the geometric properties of the global region (such as the shape of the perimeter and the geometrical center of mass). Information about precise location and region may be separately encoded, and when remembering the object both types of information could be retrieved and, together, influence our judgment. Thus, our memory of space will reflect a "blend" of the two types of spatial information.

Huttenlocher, Hedges, and Duncan (1991) have suggested that spatial perception and memory are akin to categorization, since "prototypical" spatial positions (which best capture the pattern of values encompassed by

The authors are grateful to Raghad Zaiyouna for her testing of the subjects and to the editor and referees for their thoughtful criticisms. We also thank Stephen Kosslyn for comments and suggestions on the first draft of the manuscript. Correspondence may be directed to B. Laeng, Department of Psychology, University of Tromsø, N-9037 Tromsø, Norway

—Accepted by previous editor, Geoffrey R. Loftus a category of space or region) are encoded as relevant information about where objects are in space. According to these authors, there are two ways of representing memories of locations: (1) in terms of the metrics (where, exactly), and (2) in terms of the categorical location (where, in relation to classes of locations corresponding to parsed regions of space).

One possibility is that, in general, metric indices of the precise location are less clearly remembered/perceived than categorical indices, and that, therefore, the latter information is used only once precision is no longer available. Another possibility is that our judgment may always be under the influence of both types of memories. Huttenlocher et al. (1991) have shown that subjects could make precise estimates of single locations but that, at the same time, these were clearly biased toward some prototypi$\mathrm{cal} /$ canonical locations within regions of space. These effects were demonstrated with a simple paradigm: Subjects viewed a small dot inside a circle that would appear in several angular and radial positions across trials. If both memory strategies were interacting in parallel, one would expect to see systematic errors of memory location toward some categorical feature of the area within which the dot was located. Indeed, the dot's positions were regressed toward the center-of-mass positions of the quadrants formed by the (invisible) vertical and horizontal diameters. This finding suggested that the subjects were memorizing regions of the circle containing the dot and using the prototypical central position within this region as a reference point when making their estimates. A remarkable aspect of these findings is that no subdivision of the circles into quadrants or any other subregion was provided; subjects appeared to structure the (objectively featureless) circle into quadrants and store this spatial information. 
Interestingly, about a century ago, Jastrow (1893) showed that memory for angles formed by two connected lines was biased toward angular canonical positions (i.e., the angles of the horizon and the vertical meridian and of their two intermediate diagonals). He concluded that the perception of angles (or position of lines) took place by reference to an ideal vertical and horizontal. Much more recently, Franklin, Henkel, and Zangas (1995) have attempted to extend Huttenlocher et al.'s (1991) category model to extrapersonal space in general, and have suggested that subjects impose a spatial framework of egocentric axes and that the asymmetries between the front/ back or left/right directions determine how the spatial locations of objects are encoded and remembered.

In this study, we present two experiments based on Huttenlocher et al.'s (1991) paradigm. In our first experiment, we attempted to replicate their original findings with the dot relocation task. A circle was presented for $1 \mathrm{sec}$ on the computer screen in free vision. The circle contained a dot appearing across different trials in varying angular and radial locations. After its disappearance, the subjects were asked to relocate the dot on the screen by using the mouse.

In the second experiment, we tested the hypothesis that spatial categories were better encoded by the left hemisphere (LH) than by the right hemisphere (RH). Kosslyn (1987) had proposed that the LH was specialized for categorical spatial information (e.g., qualitatively different spatial relations like above/below, left/right, inside/outside, etc.), possibly as a reflection of this hemisphere's specialization for language. A few studies have brought empirical support to this hypothesis by showing that performance in tasks requiring judgments about spatial categories is best after right visual field presentations of the stimuli (e.g., Hellige \& Michimata, 1989; Kosslyn et al., 1989; Laeng \& Peters, 1995) and that it depends on the activation of areas of the LH (as shown by PET scans; Kosslyn, 1994) or their normal functioning (as shown by deficits of brain-damaged patients; Laeng, 1994).

To further test Kosslyn's (1987) hypothesis, we modified the task used in the first experiment and lateralized tachistoscopically the same stimuli so as to give one hemisphere preferential access to the visual information. We expected that regressions toward prototypical positions within spatial categories (i.e., quadrants) would be greater after presentation to the right visual field (i.e., LH) than after presentation to the left visual field (i.e., the RH).

\section{EXPERIMENT 1}

This experiment was intended to replicate the basic findings of Huttenlocher et al. (1991). We expected that (1) angular differences of estimates of the location of a dot in a circle would regress reliably to the center of mass of each quadrant (i.e., $45^{\circ}$ of angle) and that (2) radial differences would regress toward one of the radial centraltendency values (i.e., either the average radial location of the stimuli, 0.6 radius, or the middlemost radial value that contains half the area of the full circle, 0.7 radius).

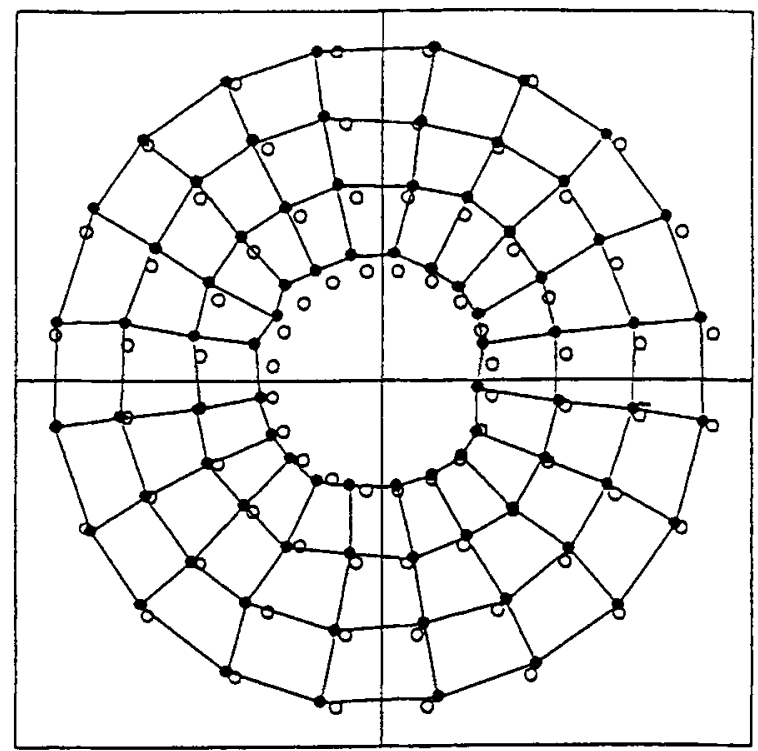

Figure 1. Experiment 1: Average response locations (black dots) for each stimulus location (white dots).

\section{Method}

Subjects. Right-handed volunteer participants, as classified by hand-preference questionnaire (cf. Laeng \& Peters, 1995), were recruited from the undergraduate population at the University of Guelph in Ontario. Nine subjects were male and 10 were female, and they did not differ in either age or handedness.

Apparatus. Stimuli were displayed on a 15-in. diagonal, color flat screen monitor with $640 \times 480$ pixels connected to a 486 IBM computer, using an ATI Mach 32 graphics card. Custom-made software controlled stimuli presentations and response records.

Procedures. A computer screen was located $42 \mathrm{~cm}$ from the chinrest on which the participant's head rested. A 1-mm black dot would then appear within a black 10.2-cm-diam circle on a white monitor screen. The dots' position varied accordirig to a 4 (quadrant) $\times 5$ (angle within quadrant) $\times 4$ (radial location within angle) design. The four possible radial positions were at $0.3,0.5,0.7$, and 0.9 of the radius from the center of the circle (i.e., 1.53-, 2.55-, 3.57-, and $4.59-\mathrm{cm}$ distances from center). The five different angular placements were at $8^{\circ}, 26.5^{\circ}, 45^{\circ}, 63.5^{\circ}$, and $82^{\circ}$ of each quadrant. Thus, there were 20 possible dot locations in a quadrant and 80 possible dot locations in the entire circle. A dot appeared only once in one of the 80 possible locations and in a different randomized order for each subject. There were no practice trials.

A trial began with the presentation of a small fixation cross centered on a blank screen. The subject would then press the mouse button onto the central point. This procedure had the advantage of forcing the subject to fixate the center of the screen at the beginning of each trial and of providing an identical starting point from which the mouse would be moved when making the estimate. The mouse click caused the instantaneous appearance of a circle (centered on the fixation point) containing one stimulus dot. The display remained on the screen for $1 \mathrm{sec}$. Then the circle and the stimulus dot disappeared and, after a delay of $1 \mathrm{sec}$, a circle-identical to the original one but empty of dots--appeared on the screen in the same position. As accurately and as quickly as possible, the subject was to click the mouse in the location where the stimulus dot had been seen.

\section{Results}

Angular differences. First, we calculated a simple regression of the angular differences (expressed as the dif- 
Table 1

Experiment 1: Linear Regression Functions for Angular Differences in Each Quadrant

\begin{tabular}{lcccc}
\hline \multicolumn{1}{c}{ Quadrant } & $\begin{array}{c}\text { Slope Coefficient } \\
\text { (and } S E)\end{array}$ & $t(18)^{*}$ & $p<*$ & $\begin{array}{c}\text { Intercept } \\
\text { (and } S E)\end{array}$ \\
\hline (A) Upper left $\left(0^{\circ}-90^{\circ}\right)$ & $0.14(0.01)$ & 17.4 & .0001 & $-6.0(0.4)$ \\
(B) Upper right $\left(90^{\circ}-180^{\circ}\right)$ & $0.08(0.01)$ & 8.4 & .0001 & $-0.4(0.4)$ \\
(C) Lower right $\left(180^{\circ}-270^{\circ}\right)$ & $0.01(0.01)$ & 1.2 & .15 & $-1.7(0.5)$ \\
(D) Lower left $\left(270^{\circ}-360^{\circ}\right)$ & $0.01(0.01)$ & 9.7 & .0001 & $-8.4(0.5)$ \\
\hline
\end{tabular}

Note--Letters A-D correspond to panels in Figure 2. ${ }^{*} t$ tests of significance of the slope coefficients.

ference between stimulus position and subject's response position) over stimuli's angular positions collapsed over all quadrants (e.g., $135^{\circ}, 225^{\circ}$, and $315^{\circ}$ were relabeled as $45^{\circ}$ ) and over all subjects. Estimates regressed positively [slope coefficient $=0.079, S E=0.01, t(18)=15.6$, $p<.0001]$, and the zero-bias position approached a central angular position of the quadrant $(x$-axis intercept $=$ $\left.52^{\circ}, S D=25\right)$, which, in fact, was not reliably different from the $45^{\circ}$ position $[t(18)=1.2, p<.1]$.

However, as Figure 1 illustrates, the mean response locations (black dots) relative to the stimulus locations (white dots) seemed to vary considerably across quad-
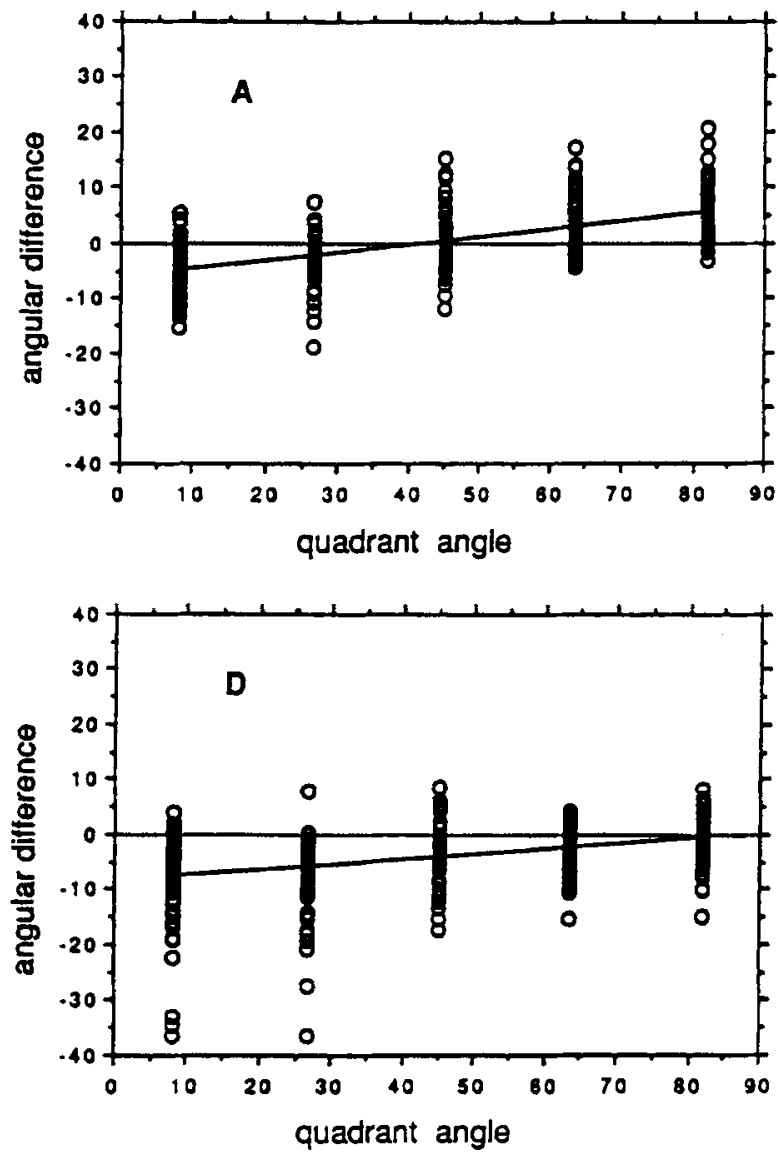

rants. Separate regression analysis for each quadrant, collapsed over subjects, revealed a somewhat unexpected pattern of biases: The upper left quadrant (see Table 1 and Figure 2A) showed a reliable bias toward $42^{\circ}(S D=$ 25 ), a position central to the quadrant and not reliably different from $45^{\circ}\left[t(18)=0.52^{\circ}, p<.25\right]$. The opposite, lower right quadrant (see Table 1 and Figure $2 \mathrm{C}$ ) showed no significant bias. The other two quadrants (i.e., the upper right, Figure 2B, and the lower left one, Figure 2D) showed reliable regressions, but their biases were, respectively, toward the $96^{\circ}(S D=25)$ and $355^{\circ}(S D=25)$ positions, that is, positions that did not differ reliably
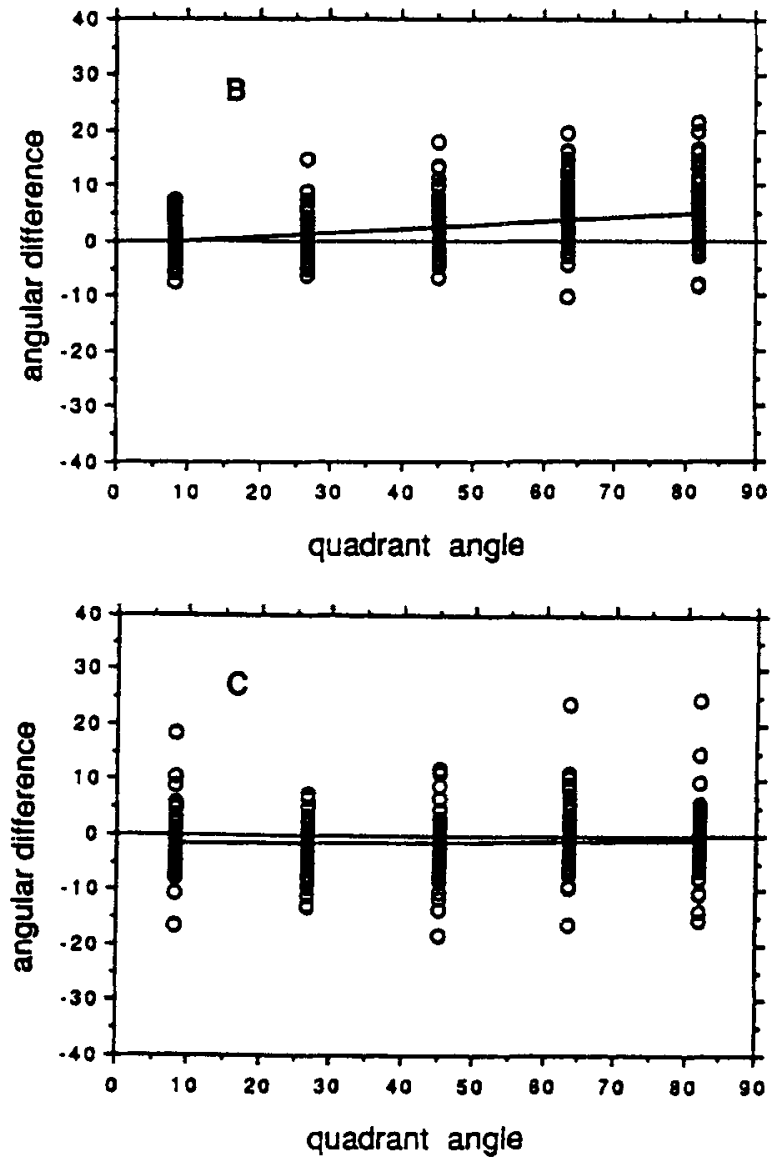

Figure 2. Experiment 1: Linear regressions of all angular differences (angular location of stimulus minus angular location of response) over angular locations of stimuli for each quadrant, uncollapsed over subjects. Panel $A=$ upper left quadrant: panel $B=$ upper right quadrant; panel $C=$ lower right quadrant; panel $D=$ lower left quadrant. 
Table 2

Experiment 1: Linear Regression Functions for Radial Differences in Each Quadrant

\begin{tabular}{lcclr}
\hline \multicolumn{1}{c}{ Quadrant } & $\begin{array}{c}\text { Slope Coefficient } \\
\text { (and } S E)\end{array}$ & $t(18)^{*}$ & $p<*$ & $\begin{array}{r}\text { Intercept } \\
\text { (and } S E)\end{array}$ \\
\hline (A) Upper left $\left(0^{\circ}-90^{\circ}\right)$ & $0.07(0.02)$ & 6.1 & .0001 & $-0.08(0.01)$ \\
(B) Upper right $\left(90^{\circ}-180^{\circ}\right)$ & $0.04(0.01)$ & 4.2 & .0001 & $-0.04(0.01)$ \\
(C) Lower right $\left(180^{\circ}-270^{\circ}\right)$ & $0.02(0.01)$ & 1.9 & .05 & $0.01(0.01)$ \\
(D) Lower left $\left(270^{\circ}-360^{\circ}\right)$ & $0.06(0.02)$ & 4.9 & .0001 & $-0.05(0.01)$ \\
\hline
\end{tabular}

Note--Letters A-D correspond to panels in Figure $3 .{ }^{*} t$ tests of significance of the slope coefficients.

from the two quadrants' boundaries with the upper left quadrant.

Radial differences. As in the analyses described above, we first calculated a simple regression of the radial differences (between stimulus position and subject's response position) over the radial position of each stimulus (collapsing data over quadrants and all subjects). This analysis revealed a statistically reliable [slope coefficient $=$ $0.05, S E=0.01, t(18)=7.9, p<.0001]$ positive slope toward 0.77 radius $(S D=0.24)$ - that is, a value not reliably different from the radial middlemost value $(0.70)$ $[t(18)=1.2, p<.25]$.
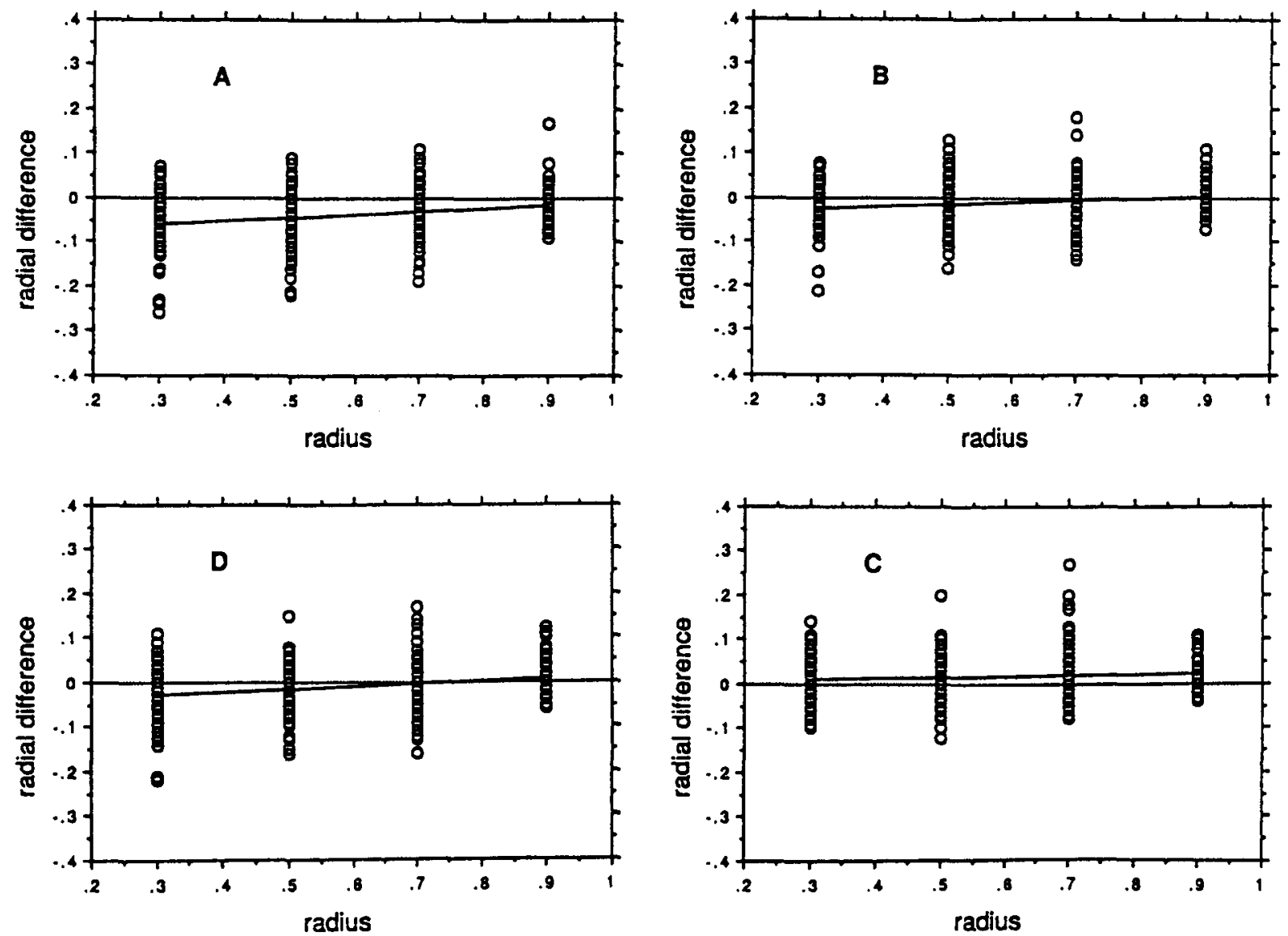

Figure 3. Experiment 1: Linear regressions of all radial differences (radial location of stimulus minus angular location of response) over radial locations of stimuli for each quadrant, uncollapsed over subjects. Panel $A=$ upper left quadrant; panel $B=$ upper right quadrant; panel $\mathrm{C}=$ lower right quadrant; panel $\mathrm{D}=$ lower left quadrant.
Separate regression analyses for each quadrant's data collapsed over subjects revealed statistically reliable regressions for all quadrants (see Table 2). In the upper left quadrant, the radial estimates appeared displaced toward 1.00 radius $(S D=0.24)$ or the circumference (see Figure $3 \mathrm{~A}$ ). On the contrary, in the opposite quadrant (see Figure $3 \mathrm{C}$ ), the radial estimates appeared displaced away from the circumference and to a position (radius $=0.2$, $S D=0.24)$ closer to the center of the circle but reliably different from this position as well $[t(18)=3.57, p<.005]$. In the other two quadrants (see Figures $3 B$ and $3 D$ ), the estimates were displaced toward 0.8 radius $(S D=0.24)$ -

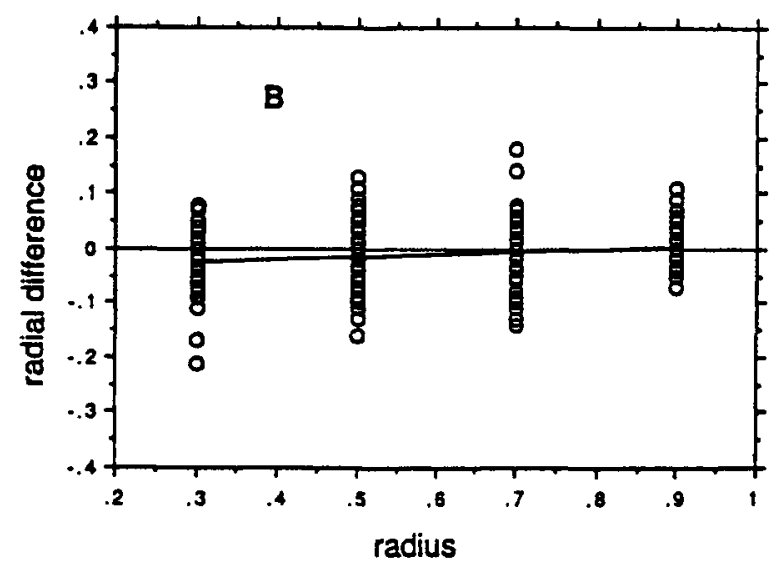


Table 3

Experiment 2: Linear Regression Functions for Angular Differences in Each Quadrant

\begin{tabular}{lcccr}
\hline \multicolumn{1}{c}{ Quadrant } & $\begin{array}{c}\text { Slope Coefficient } \\
\text { (and } S E)\end{array}$ & $t(18)^{*}$ & $p<*$ & $\begin{array}{r}\text { Intercept } \\
\text { (and } S E \text { ) }\end{array}$ \\
\cline { 1 - 3 } & Left Visual Field & & & \\
(A) Upper left $\left(0^{\circ}-90^{\circ}\right)$ & $0.20(0.02)$ & 13.3 & .0001 & $-11.2(0.8)$ \\
(B) Upper right $\left(90^{\circ}-180^{\circ}\right)$ & $0.09(0.02)$ & 5.8 & .0001 & $-1.7(0.8)$ \\
(C) Lower right $\left(180^{\circ}-270^{\circ}\right)$ & $-0.03(0.02)$ & 1.3 & .15 & $0.6(1.1)$ \\
(D) Lower left $\left(270^{\circ}-360^{\circ}\right)$ & $0.03(0.02)$ & 1.4 & .10 & $-9.6(1.0)$ \\
& Right Visual Field & & & \\
(A) Upper left $\left(0^{\circ}-90^{\circ}\right)$ & $0.18(0.02)$ & 10.0 & .0001 & $-4.5(0.9)$ \\
(B) Upper right $\left(90^{\circ}-180^{\circ}\right)$ & $0.08(0.02)$ & 4.8 & .0001 & $-2.5(0.1)$ \\
(C) Lower right $\left(180^{\circ}-270^{\circ}\right)$ & $0.01(0.02)$ & 0.6 & .60 & $-4.6(0.9)$ \\
(D) Lower left $\left(270^{\circ}-360^{\circ}\right)$ & $0.09(0.02)$ & 5.1 & .0001 & $-7.5(0.9)$ \\
\hline
\end{tabular}

Note-Letters A-D correspond to panels in Figures 5 and $6 . *^{*} t$ tests of significance of the slope coefficients.

that is, a position not reliably different from the radial middlemost value $(0.7$ radius $)[t(18)=1.7, p<.06]$ but reliably distant from the circumference $[t(18)=3.6, p<$ .005]. This pattern of displacement of the radial values of estimates clearly revealed a general shift of responses in the direction of the upper left arc of the circle.

\section{Discussion}

The overall pattern of results was consistent with Huttenlocher et al.'s (1991) findings of a regression of estimates of points toward internal locations of parsed regions. Indeed, when the angular values of estimates of a dot's position inside a circle were collapsed over all quadrant angles, these regressed toward the center of the quadrant. Radial locations, instead, were biased, on average, toward 0.8 radius, thus a value closer to the middlemost radial value than to the circumference. These systematic errors show that responses tend to group around middle positions on the middle diagonals of quadrants. Thus, we conclude that biases in estimates occur toward ideal positions within the regions defined by boundaries formed by the horizontal and vertical axes.

However, the above conclusions cannot entirely account for the pattern of responses that we observed; in fact, when the estimates were examined separately for each quadrant's locations, both the angular and radial values appeared to be displaced toward different positions in each quadrant (Figures 2 and 3). Nevertheless, the latter types of displacements obeyed a very orderly pattern (see Figure 1), and could be characterized as an overall shift toward the upper left arc of the circle. This was best exemplified by the strong bias of radial values toward the circumference ( 1.0 radius) in the upper left quadrant versus a bias toward 0.2 radius in the opposite, lower right quadrant.
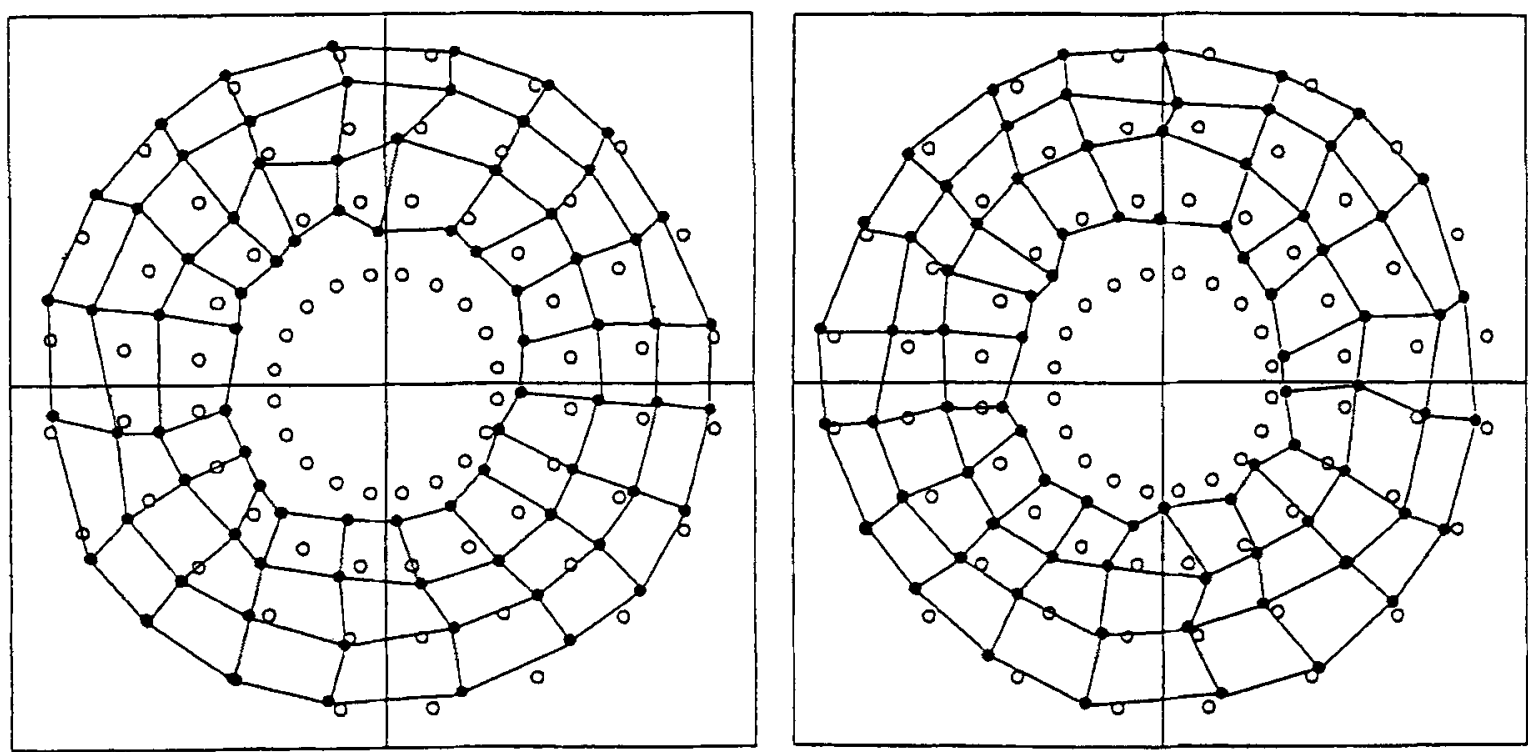

Figure 4. Experiment 2: Average response location (black dots) for each stimulus location (white dots) in the left visual field (left graph) and right visual field (right graph). 

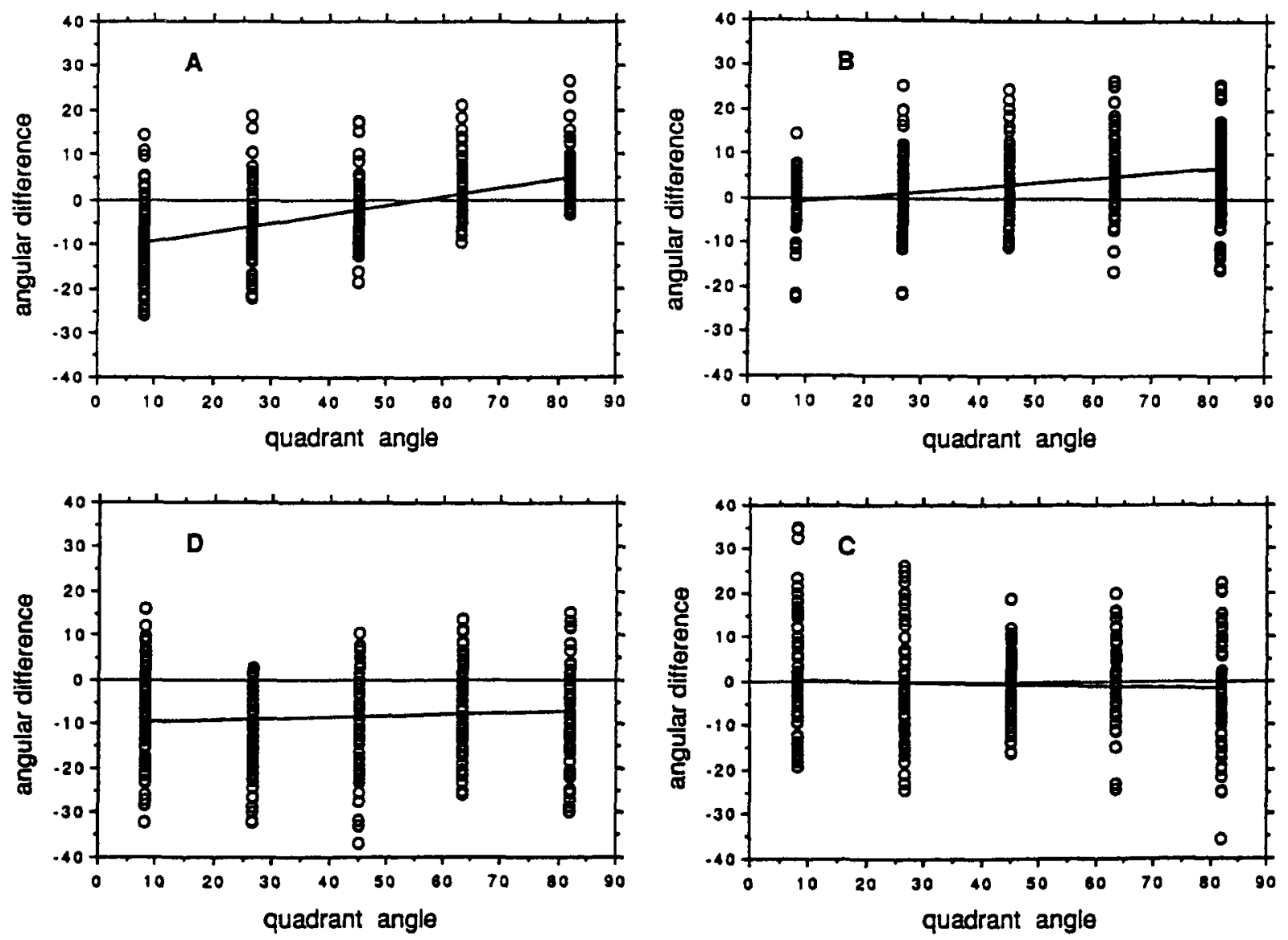

Figure 5. Experiment 2, left visual field: Linear regressions of all angular differences (angular location of stimulus minus angular location of response) over angular locations of stimuli for each quadrant, uncollapsed over subjects. Panel $A=$ upper left quadrant; panel $B=$ upper right quadrant; panel $C=$ lower right quadrant; panel $D=$ lower left quadrant.

Thus, we conclude that superimposed on the original effect described by Huttenlocher et al. (1991), there was also another, more general shift of all responses toward the upper left direction.

This bias toward the left of the display is reminiscent of other biases shown by normal subjects when making perceptual evaluations of length or position. Biases are observed especially in spatial tasks, such as line bisection, in which patients with (typically) unilateral posterior lesions of the brain show "dramatic" systematic errors. For example, when normal subjects are asked to bisect lines equally (e.g., Bradshaw, Nathan, Nettleton, Wilson, \& Pierson, 1987; Milner, Brechmann, \& Pagliarini, 1992; or-in the tactile modality-rods; e.g., Bowers \& Heilman, 1980; Laeng, Buchtel, \& Butter, 1996), they tend to err to the left of the actual physical center. This bias is typically explained by either a tendency to deploy attention to the left of a display (Bowers \& Heilman, 1980) or by a systematic (from left to right) scanning of the linear extent of the stimulus (see, e.g., Manning, Halligan, \& Marshall, 1990). It is possible that circular stimuli are not scanned randomly; consequently, internal positions close to the focus of attention may benefit in processing, whereas locations far from the starting point of ocular/attentional scanning will pay a cost (depending also on the duration of the display).

Finally, we need to consider the possibility that the regression of radial values of estimates in an outward direction (see Figure $3 \mathrm{~A}$ ), instead of toward the quadrant's center, may be an artifact resulting from the fact that responses cannot exceed the natural boundary of the circumference. Since radial values of estimates can never be greater than a specific upper value (i.e., 1.0 radius), this can result in a systematic and asymmetric (but artifactual) reduction of the errors' spread, which increases proportionally with the vicinity of the dot stimulus to the circumference (see how errors' spread reduces at 0.9 radius in Figures 3A, 3B, 3C, and 3D). Nevertheless, it would seem that radial estimates were also affected by counteracting biases toward the interior of the quadrant, since the zero bias in two quadrants (see Figures 3B, 3C, and 3D) was closer to the middlemost radial value, 0.7 radius, 

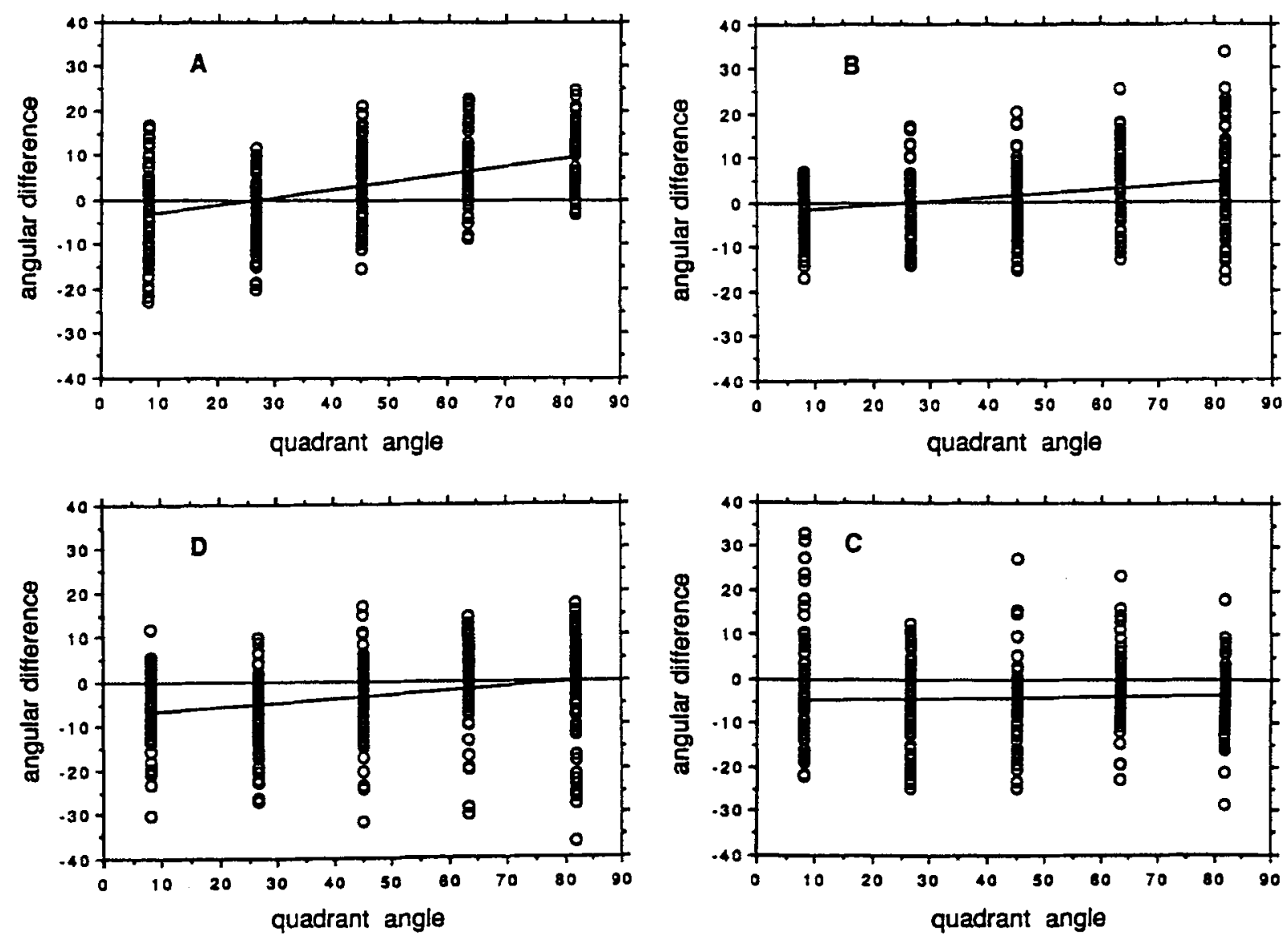

Figure 6. Experiment 2, right visual field: Linear regressions of all angular differences (angular location of stimulus minus angular location of response) over angular locations of stimuli for each quadrant, uncollapsed over subjects. Panel $A=$ upper left quadrant; panel $B=$ upper right quadrant; panel $C=$ lower right quadrant; panel $D=$ lower left quadrant.

than to the circumference and in one quadrant was, in fact, shifted toward the center of the circle.

\section{EXPERIMENT 2}

The aim of the second experiment was to show that the within-quadrant biases seen in Huttenlocher et al.
(1991) and in the results above are dependent on the superior ability of the LH in processing categorical spatial relations. Thus, we expected that the biases toward prototypical locations of spatial regions would be greater after right visual field (RVF) than after left visual field (LVF) presentations of the same stimuli used in the preceding experiments.

Table 4

Experiment 2. Linear Regression Functions for Radial Differences in Each Quadrant

\begin{tabular}{|c|c|c|c|c|}
\hline Quadrant & $\begin{array}{c}\text { Slope Coefficient } \\
\text { (and } S E \text { ) }\end{array}$ & $t(18)^{*}$ & $p<*$ & $\begin{array}{l}\text { Intercept } \\
\text { (and } S E \text { ) }\end{array}$ \\
\hline \multicolumn{5}{|c|}{ Left Visual Field } \\
\hline (A) Upper left $\left(0^{\circ}-90^{\circ}\right)$ & $0.23(0.02)$ & 11.8 & .0001 & $-0.3(0.01)$ \\
\hline (B) Upper right $\left(90^{\circ}-180^{\circ}\right)$ & $0.20(0.02)$ & 10.2 & .0001 & $-0.2(0.01)$ \\
\hline (C) Lower right $\left(180^{\circ}-270^{\circ}\right)$ & $0.18(0.02)$ & 8.2 & .0001 & $-0.1(0.01)$ \\
\hline (D) Lower left $\left(270^{\circ}-360^{\circ}\right)$ & $0.16(0.02)$ & 7.4 & .0001 & $-0.2(0.02)$ \\
\hline \multicolumn{5}{|c|}{ Right Visual Field } \\
\hline (A) Upper left $\left(0^{\circ}-90^{\circ}\right)$ & $0.21(0.02)$ & 10.2 & .0001 & $-0.2(0.02)$ \\
\hline (B) Upper right $\left(90^{\circ}-180^{\circ}\right)$ & $0.21(0.02)$ & 10.1 & .0001 & $-0.2(0.01)$ \\
\hline (C) Lower right $\left(180^{\circ}-270^{\circ}\right)$ & $0.22(0.02)$ & 12.6 & .0001 & $-0.1(0.01)$ \\
\hline (D) Lower left $\left(270^{\circ}-360^{\circ}\right)$ & $0.16(0.02)$ & 8.9 & .0001 & $-0.2(0.01)$ \\
\hline
\end{tabular}

Note-Letters A-D correspond to panels in Figures 7 and $8 . \quad *_{t}$ tests of significance of the slope coefficients. 

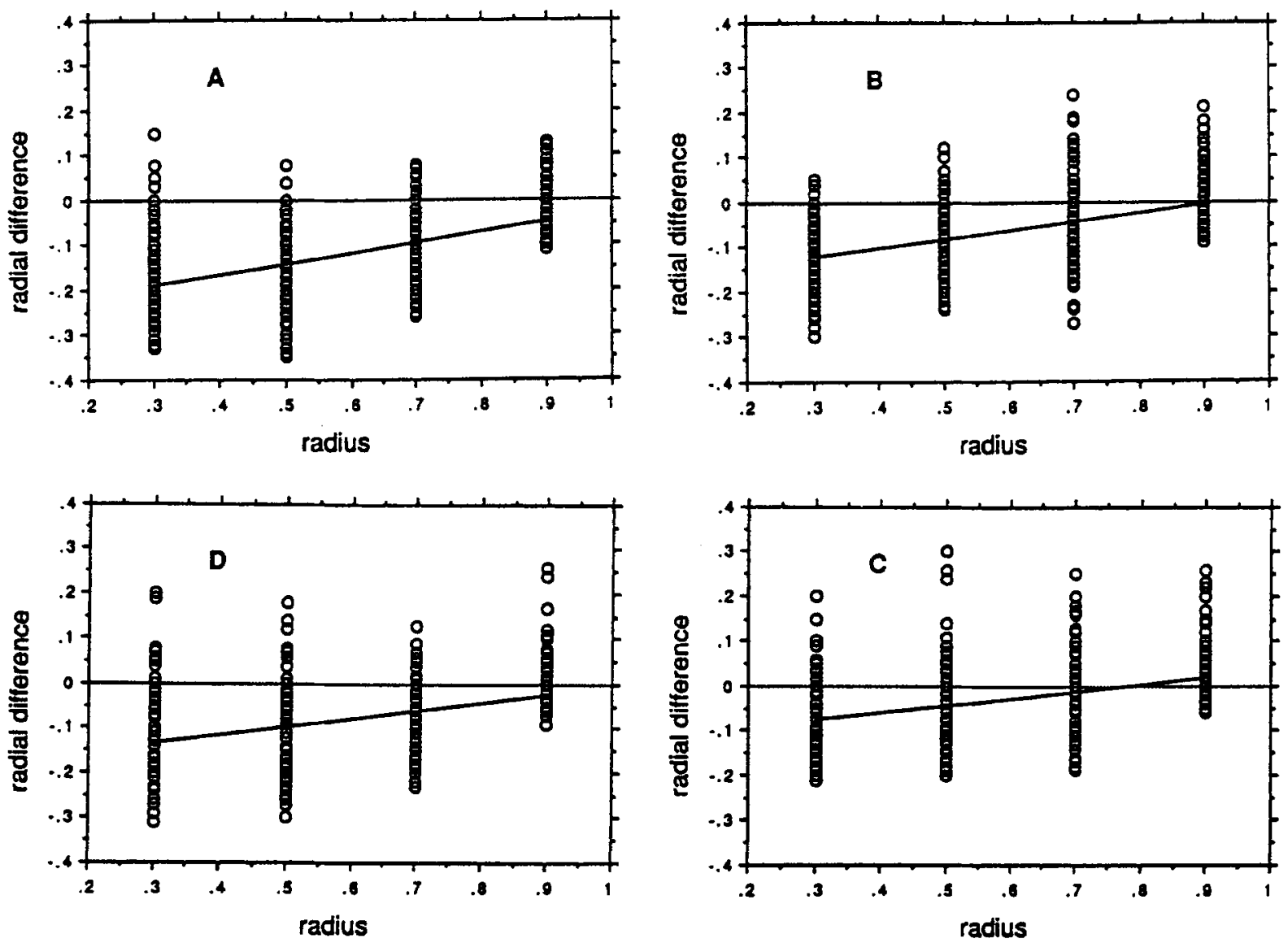

Figure 7. Experiment 2, left visual field: Linear regressions of all radial differences (radial location of stimulus minus angular location of response) over radial locations of stimuli for each quadrant, uncollapsed over subjects. Panel $A=$ upper left quadrant; panel $B=$ upper right quadrant; panel $C=$ lower right quadrant; panel $D=$ lower left quadrant.

\section{Method}

After completion of the first experiment, the procedures of a second experiment were explained to the same subjects. The subjects were told that the circle would now appear on either the left or the right of the fixation point (the center of the circle was now located at a distance of $11.5 \mathrm{~cm}$ from the fixation point) and that once the empty circle was again visible (after $1 \mathrm{sec}$ ) in its original location, they were to indicate, by pressing the mouse button, the dot's location on the screen. The subjects were reminded that they should respond as quickly and accurately as possible.

At the beginning of each trial, only the central fixation point was visible. The subjects were told to move the cursor on the small cross at the center of the screen and to press the mouse button while maintaining fixation. The buttonpress initiated a 85 -msec lateralized exposure of the circle with one stimulus dot inside it. Thus, presentation time was comfortably below the estimated latency of a saccadic eye movement (see Woodworth \& Schlosberg, 1954), which would guarantee that the stimuli would be seen in one visual field only and disappear before the subject abandoned fixation. The left or right position of the circle was varied in a pseudorandom sequence (i.e., no more than three subsequent presentations in the same lateral field were allowed), which was different for each subject. In all, there were 80 presentations on the left and 80 presentations on the right of the fixation point. Locations of the dots within each circle were the same as in Experiment 1.

\section{Results}

Angular differences. Following the same procedures of data analysis used in Experiment 1, we first calculated two separate simple regressions, one for each visual hemifield condition (LVF, RVF), of the angular differences over the angular positions of stimuli (collapsed over all quadrants and over all subjects). As predicted, estimates regressed positively in both conditions [LVF, slope = $0.075, S E=0.01, t(18)=7.24, p<.0001 ; \mathrm{RVF}$, slope $=$ $0.090, S E=0.01, t(18)=9.44, p<.0001]$. However, the zero-bias value approached the central angular position of the quadrant only with the RVF presentations $\left[x\right.$-axis intercept $\left.=53^{\circ}, S D=25 ; t(18)=1.3, p<.10\right]$ and not with the ones in the LVF $\left[x\right.$-axis intercept $=67^{\circ}$, $S D=25, t(18)=3.8, p<.005]$.

To test for differences in the regression slopes of the two visual fields, we performed a repeated measures analysis 

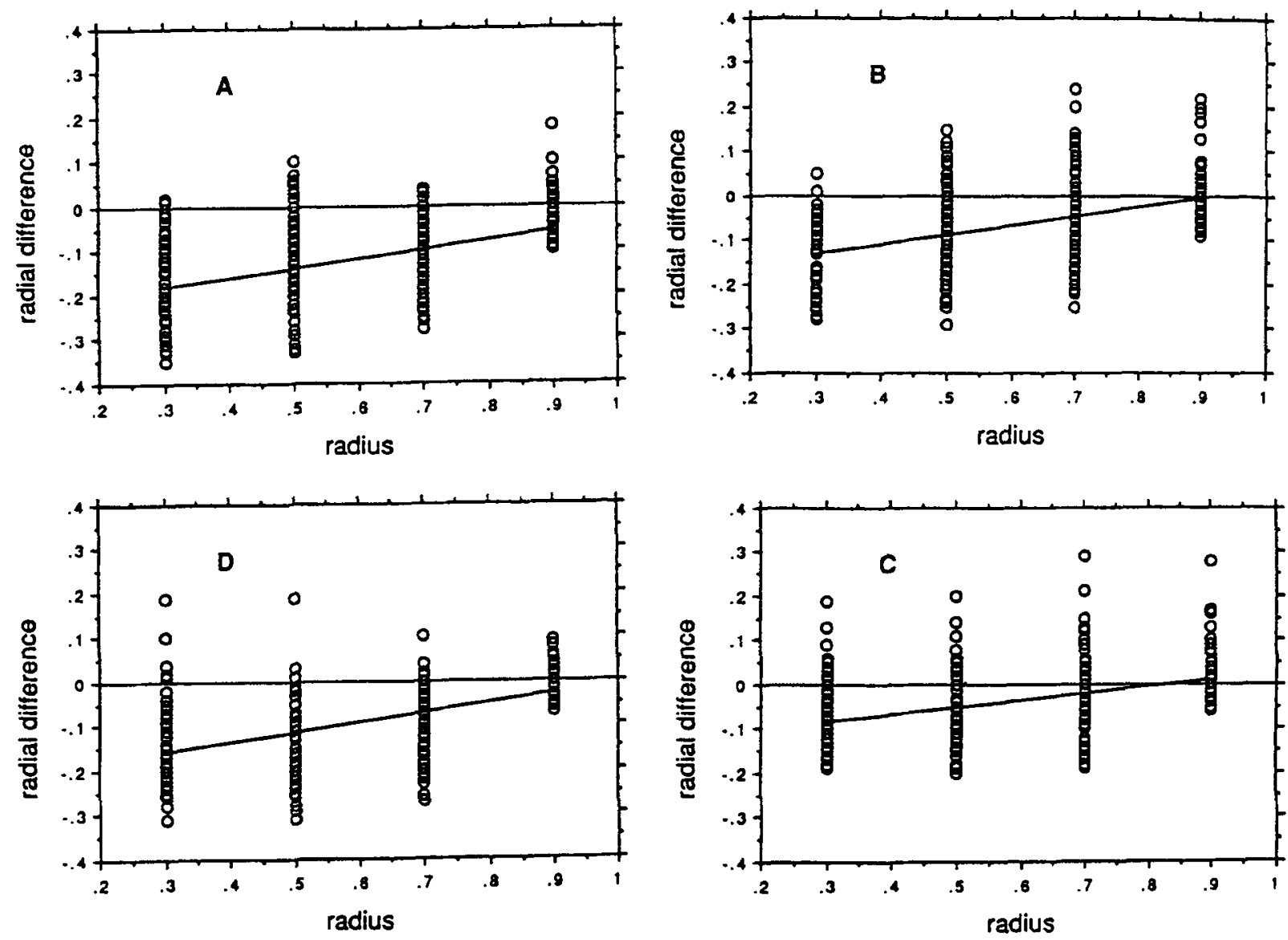

Figure 8. Experiment 2, right visual field: Linear regressions of all radial differences (radial location of stimulus minus angular location of response) over radial locations of stimuli for each quadrant, uncollapsed over subjects. Panel $A=$ upper left quadrant; panel $B=$ upper right quadrant; panel $C=$ lower right quadrant; panel $D=$ lower left quadrant.

of variance on each subject's slope coefficients in each quadrant as the dependent variable and the visual hemifield (LVF, RVF) and quadrant (upper right, lower right, lower left, and upper left) as the within-subjects factors. This analysis revealed reliable differences between hemifields [LVF, mean slope $=0.068, S D=0.12 ; \mathrm{RVF}$, mean slope $=0.102, S D=0.14 ; F(1,18)=5.1, p<.03]$. There were also reliable differences between quadrants [upper right quadrant, mean slope $=0.107, S D=0.15$; lower right quadrant, mean slope $=-0.01, S D=0.10$; lower left quadrant, mean slope $=0.065, S D=0.11$; upper left quadrant, mean slope $=0.179, S D=0.10 ; F(3,54)=$ $21.5, p<.0001]$ but no reliable interaction between hemifields and quadrants $[F(3,54)=0.4, p<.75]$.

Separate regression analyses for each hemifield's quadrant data, collapsed over subjects, revealed a very similar pattern of biases, as seen in the previous experiment, for the central (bilateral/nontachistoscopic) condition (see Figure 4 and Table 3 ). The upper left quadrants (Figures $5 \mathrm{~A}$ and $6 \mathrm{~A}$ ) showed a bias toward positions (LVF, $\left.56^{\circ}, S D=25 ; \mathrm{RVF}, 26^{\circ}, S D=25\right)$ intermediate of the center of the quadrant $\left[45^{\circ}\right.$; LVF, $t(18)=1.9, p<.05$; RVF, $t(18)=3.3, p<.005]$ and one of the quadrant's bound- aries [LVF, $t(18)=11.8, p<.0001 ; \mathrm{RVF}, t(18)=4.5$, $p<.0001]$. The opposite, lower right quadrants showed no significant biases (Figures $5 \mathrm{C}$ and $6 \mathrm{C}$ ) in either field condition. The upper right quadrants showed reliable regressions in both fields (Table 3; Figures $5 \mathrm{~B}$ and $6 \mathrm{~B}$ ). In the LVF, there was a bias toward the $92^{\circ}(S D=25)$ position, that is, a location not reliably different from this quadrant's boundary with the upper left quadrant [i.e., $\left.90^{\circ} ; t(18)=0.34\right]$. In the RVF, there was a bias toward a position $\left(116^{\circ}, S D=25\right)$ intermediate of the center of the quadrant $\left[135^{\circ} ; t(18)=3.3, p<.005\right]$ and the quadrant's boundary with the upper left quadrant $[t(18)=$ $3.8, p<.005]$. For the lower left quadrants (Figures 5D and $6 \mathrm{D}$ ), only the RVF condition showed a reliable bias toward $351^{\circ}(S D=25)$, or a value not significantly distant from the $360^{\circ}$ boundary $[t(18)=1.5, p<.10]$. There was no reliable regression of estimates in the LVF (see Table 3).

Radial differences. We first performed two separate, one for each hemifield, simple regressions of the radial differences (between stimulus position and subject's response position) over the radial position of each stimulus (collapsing data over quadrants and subjects). These 
analyses revealed statistically reliable positive slopes [LVF, slope coefficient $=0.19, S E=0.011, t(18)=17.66$, $p<.0001$; RVF, slope coefficient $=0.205, S E=0.020$, $t(18)=10.17, p<.0001]$ toward the circumference in both the LVF ( 0.9 radius, $S D=0.27)$ and RVF (1.0 radius, $S D=0.28$ ) conditions.

A repeated measures analysis of variance, in which each subject's four slope coefficients (for each of the regressions in each quadrant) were used as the dependent variable and hemifield (LVF, RVF) and quadrant (upper right, lower right, lower left, and upper left) as the withinsubjects factors, revealed no reliable differences between hemifields $[F(1,18)=0.24, p<.63]$, quadrants $[F(3,54)=$ $2.116, p<.11]$, or the interaction of these two factors $[F(3,54)=0.52, p<.50]$.

Separate regression analyses of the data for each quadrant (collapsed over subjects) revealed statistically reliable regression effects for each quadrant and for both hemifields (see Table 4 and Figures 7 and 8). Again, we observed a generic shift of radial estimates toward the upper left direction (see also Figure 4). This was demonstrated by a bias toward the most outward radial position (1.0 radius) of the upper left quadrant (see Figures 7A and 8A); in the opposite, lower right quadrant, in both the LVF and RVF (see Figures 7C and $8 \mathrm{C}$ ), there was a bias closer to central values [0.8 radius; i.e., no significant distance from the radial middlemost value: $t(18)=1.6, p<.10$ ].

\section{Discussion}

We found that angular biases were greater after tachistoscopic RVF than after LVF presentations of the stimuli; that is, the slopes of the regression lines of the angular biases over angular position were steeper in the RVF than in the LVF. This finding suggests that categorical spatial information has a stronger influence in the LH than in the RH, which is consistent with Kosslyn's (1987) hypothesis that the LH is specialized in processing categorical spatial location.

However, we again observed great variations in the subjects' performance across quadrants (cf. Huttenlocher et al., 1991). It is possible that our subjects were using other landmarks in addition to the prototypical centers of the quadrants; therefore, estimates may reflect the combined effect of several contextual cues. One possibility, strongly suggested by a shift of estimates toward a left and upper direction, is that subjects used the upper left arc of the circle as a reference point. Nevertheless, it remains unclear as to why our subjects would have used visual cues that were so different from those used by Huttenlocher et al.'s subjects.

The possibility that the bias toward the upper left arc might have resulted from a motor, rather than a perceptually based, directional bias can be discarded. According to this account, our right-handed subjects may have been less precise in moving the cursor toward the upper left than they were in moving it in other directions. However, this cannot explain why there was still a shift of es- timates toward the upper left arc in the RVF, since a movement toward that quadrant required, in this condition, moving the mouse from the center of the screen toward the upper right direction.

\section{GENERAL DISCUSSION}

The observation of a bias toward a position interior to a spatial region, as opposed to a randomly distributed difference around the true location of a stimulus, can be taken as evidence (cf. Huttenlocher et al., 1991) that estimation of location is influenced by the parsing of a visual display into spatial regions. More specifically, spatial memory is biased toward salient features, landmarks, and geometrical centers of these regions. Clearly, our findings supported the more specific hypothesis that recollections of the angular and/or radial location of a dot viewed within a circle can be biased toward locations internal to regions of the circle. Such an effect was captured by the linear regression functions of angular and radial differences toward an internal value of each quadrant.

We conclude that our findings support the idea that imagined, illusory, categorical boundaries and regions defined by these boundaries are encoded by the viewer and that these subjective landmarks in the display have a determinable influence on the representation of spatial location (see Bryant \& Subbiah, 1994, for a systematic study of inducing - via the use of visual cues - different subdivisions of the input in regions). Additionally, the finding of a greater bias in regressions within quadrants in the RVF than in the LVF is consistent with the view that spatial categories play a greater role in the LH than in the RH. Kosslyn (1987) has specifically proposed that the encoding of categorical spatial relations is a LH's specialization. However, this study did not address directly what specific cognitive mechanism (e.g., perceptual encoding, storage, or retrieval from memory, selection of the response) could underlie the difference between the cerebral hemispheres.

Finally, Huttenlocher et al. (1991) have proposed that the bias toward the prototype, when combined with the fine-grain memories of locations, actually improves the accuracy of estimations. In the light of the findings described in this study, this would seem to suggest that the (LH's) biasing effect emerges as a fruitful correction mechanism for estimating spatial locations with greater precision. It is possible that cooperation between the two hemispheres is more adaptive than unilateral hemispheric control across a wide range of perceptual experiences even though, in specific circumstances, it may not be the most efficient strategy (cf. Hellige, 1993).

\section{REFERENCES}

Bowers, D., \& Heilman, K. M. (1980). Pseudoneglect: Effects of hemispace on a tactile line bisection task. Neuropsychologia, 18, 491-498 Bradshaw, J. L., Nathan, G., Nettleton, N. C., Wilson, L., \& Pier- 
SON, J. (1987). Why is there a left side underestimation in rod bisection? Neuropsychologia, 25, 735-738.

Bryant, D. J., \& Subbiah, I. (1994). Subjective landmarks in perception and memory for spatial location. Canadian Journal of Experimental Psychology, 48, 119-139.

Franklin, N., Henkel, L. A., \& Zangas, T. (1995). Parsing surrounding space into regions. Memory \& Cognition, 23, 397-407.

HELLIGE, J. B. (1993). Hemispheric asymmetry: What's right and what's left. Cambridge, MA: Harvard University Press.

Hellige, J. B., \& Michimata, C. (1989). Categorization versus distance: Hemispheric differences for processing spatial information. Memory \& Cognition, 17, 770-776.

Huttenlocher, J., Hedges, L. V., \& Duncan, S. (1991). Categories and particulars: Prototype effects in estimating spatial location. Psychological Review, 98, 352-376.

JASTROW, J. (1893). On the judgment of angles and positions of lines. American Journal of Psychology, 5, 214-268.

KossLYN, S. M. (1987). Seeing and imaging in the cerebral hemispheres: A computational approach. Psychological Review, 94, 2, 148-175.

KosSLYN, S. M. (1994). Image and brain: The resolution of the imagery debate. Cambridge, MA: MIT Press.

Kosslyn, S. M., Koenig, O., Barrett, A., Cave, C. B., Tang, J., \& GABRIELI, J. D. E. (1989). Evidence for two types of spatial repre- sentations: Hemispheric specialization for categorical and coordinate relations. Journal of Experimental Psychology: Human Perception \& Performance, 15, 743-750.

LAENG, B. (1994). Lateralization of categorical and coordinate spatial functions. A study of unilateral stroke patients. Journal of Cognitive Neuroscience, 6, 189-203.

Laeng, B., Buchtel, H. A., \& Butter, C. M. (1996). Tactile rod bisection: Hemispheric activation and sex differences. Neuropsychologia, 34, 1115-1121.

Laeng, B., \& Peters, M. (1995). Cerebral lateralization for the processing of spatial coordinates and categories in left- and right-handers. Neuropsychologia, 33, 421-439.

Manning, L., Halligan, P. W., \& Marshall, J. C. (1990). Individual variation in line bisection: A study of normal subjects with application to the interpretation of visual neglect. Neuropsychologia, 28, 647-655.

Milner, A. D., Brechmann, M., \& Pagliarini, L. (1992). To halve and to halve not: An analysis of line bisection judgments in normal subjects. Neuropsychologia, 36, 515-526.

WOODWORTH, R. S., \& SCHLOSBERG, H. (1954). Experimental psychology. New York: Holt, Rinehart \& Winston.

(Manuscript received June 19, 1996; revision accepted for publication October 1, 1996.) 